RESEARCH ARTICLE

\title{
Effect of Pre-germination Technique on Enhancing Seed and Seedling Vigour in Ridge gourd (Luffa acutangla Roxb.)
}

\author{
Abinaya $K^{1 *}$, Sundareswaran $S^{2}$ and Raja $K^{1}$ \\ ${ }^{1}$ Department of Seed Science and Technology, Tamil Nadu Agricultural University, Coimbatore - 641003. \\ ${ }^{2}$ Directorate of Seed Centre, Tamil Nadu Agricultural University, Coimbatore - 641003.
}

Received : $22^{\text {nd }}$ April, 2020

Revised : $14^{\text {th }}$ May, 2020

Revised : $30^{\text {th }}$ May, 2020

Accepted : $16^{\text {th }}$ June, 2020

\begin{abstract}
Sowing pre-germinated seeds has proved to be an effective technique to obtain early emergence, optimum field stand and high germination percentage. Before sowing, the pre-germination method could be used to separate dead seed and as dead low vigor seeds to get high vigor seeds, which can be taken for sowing. With this notion, an experiment was conducted with ridge gourd seed var. PKM 1 with two factors, one as soaking duration $(6,9,12,16,20$, and $24 \mathrm{~h})$ and other as the incubation periods (1, 2, 3 and 4 days). Seeds were soaked in distilled water for the above-mentioned different durations in a 1:1 ratio on a volume basis. After a specified period of soaking, the seeds were placed in between the moist gunny bags and kept for incubation under dark conditions. Data were collected for different parameters viz., sprouting percentage, germination percentage, root length, shoot length, dry matter production and vigor index I \& II and analyzed. Results obtained from the study showed that there is a significant difference between the treatments and seeds soaked in water for $20 \mathrm{~h}$ with one-day incubation recorded the highest values in all the parameters. The seeds that were incubated without soaking performed similar to that of absolute control because of the presence of hard seed coat. Whereas, seeds that were soaked but not incubated had performed better than dry seed and only incubated seeds. This might be because, soaking seeds in water stimulates the pre-germinative metabolism, activates enzymes earlier and also releases the physical dormancy of ridge gourd seeds which makes the seed to germinate easier and faster. Hence, the study concluded that this technique might be considered as farmer's friendly method that can be used to separate live seed in a seed lot, which will be useful for producing healthy seedlings, maintaining optimum plant population and uniformity in seedling emergence of ridge gourd.
\end{abstract}

Keywords: Pre-germination; ridge gourd; soaking; incubation; sprouting percentage and seedling emergence.

\section{INTRODUCTION}

Vegetable crops comprise a huge number of species that are included as an essential component in our daily diet. Crops, which belong to the family Cucurbitaceae, are called cucurbits or gourds. Cucurbits are one of the important crops, which is under cultivation in India and all over the world. Ridge gourd (Luffa acutangla) belongs to the family Cucurbitaceae, subfamily Cucurbitoideae, Tribe Benincaseae, Subtribe Luffinae and genus Luffa (Bates, 1990). It is an annual subtropical vine vegetable that originated from tropical Asia and Africa. The genus name was derived from the product "Loofah" which is used as bathing sponges, scrubber pads, doormats, pillows, mattresses, cleaning utensils etc., Ridge gourd is commonly called as angled loofah, ribbed gourd, Chinese okra, silk squash and locally called as 'peerkan' in Tamil Nadu. It requires a temperature range between $25-30^{\circ} \mathrm{C}$ for proper germination. It grows more commonly in tropical areas and can be raised in containers for transplantation whenever necessary. The seed yields an oil which is, however bitter and toxic (Burkill, 1985; Lim, 2012a,b) and the oil content present is $26 \%$; the fatty acid composition is linoleic acid $34 \%$, oleic acid 24\%, palmitic acid 23\%, and stearic acid 10\% (Uriostegui , 2015). Luffaculin a glycoprotein extracted from the seeds, which shows abortifacient, antitumor, ribosome-inactivating and immunomodulatory activities (Fernando and Grun, 2001). The seeds possess purgative and emetic

$$
107|4-6| 119
$$


properties and it is found that they also contain a gelatinous compound called luffein.

Rapid and uniform emergence of crop plant in the field is extremely important, which is the foundation on which stand establishment and potential yield is determined. Variation in emergence can reduce crop yield. Most of the cucurbits have a hard seed coat, leading to uneven germination and irregular crop stand in the field (Bidhan, 2013). The major problems encountered during the cultivation of gourds are uneven germination, wastage of seeds, and loss of seeds due to rodents, which ultimately results in yield reduction.

To overcome these issues, the transplantation technique is used nowadays instead of direct sowing in the pit. Generally in traditional sowing, 3-5 seeds were sown per pit and then later thinning is followed to reduce the number of seedlings. Whereas in transplanting technique one healthy seedling per pit is transplanted and it is maintained properly to get optimum plant population in the field. Cucurbitaceous seeds are more sensitive to soil moisture levels therefore, field emergence in these crops has always been problematic even with seeds of high germinability (Krishnasamy, 1991). By using pre-germination technique, rapid germination and guaranteed selection of viable seeds are possible and so, it is considered as a more reliable estimate of seed requirement during sowing, which is used for optimizing seed rate (Bruggink et al., 1996).

To overcome this problem, a pre-germination technique that involves seed hydration up to the point of radicle protrusion could be practiced. The pre-germination method results in rapid and uniform seed germination by means of avoiding unpredictable environmental conditions on germination by advancing seedling emergence (Gray, 1981). Sowing of pre-germinated seeds selected based on the length of the emerged radicle can be practiced to get optimum field stand during seed production. During direct sowing of seeds in the field, there is no chance for removing the non-viable and damaged seeds, which ultimately give uneven germination leading to uneven crop stand (Bidhan, 2013). On the other hand, the pre-germinated seed has a limited shelf life and needs to be sown as soon as possible in the field or more preferably, in protray for obtaining an optimum seedling establishment.

Pre-germinated seeds were found to be superior in emergence from the soil and stand establishment. The additional advantages of the pre-germination technique include, lower seed rate, dead seeds and germinating but low vigor seeds can be discarded before sowing. Viable and high vigorous seeds were separated and used for sowing in portray, which gives healthy for transplantation. Ghate and Phatak
(1982) reported that seedlings from pre-germinated seeds were found to be heavier and highly vigorous than other seedlings. Each crop requires a critical soaking duration for inducing radicle protrusion in seeds, above, which will cause a deleterious effect on seed germination. However, the soaking duration, incubation period and the volume of water to be used are very important to get better and uniform radicle emergence. Hence, with this background, a study was conducted to know the efficacy of the pre-germination technique on seed and seedling vigor in ridge gourd (Luffa acutangula).

\section{MATERIAL AND METHODS}

The present study was conducted in the Department of Seed Science and Technology, Tamil Nadu Agricultural University, Coimbatore during 2019-2020 to find out the effect of pre-germination technique on enhancing seed and seedling vigor in ridge gourd. Genetically pure and graded ridge gourd seeds var. PKM 1 obtained from horticultural college and research institute, Periyakulam was taken as a base material for the study.

The experiment was laid out in Factorial completely randomized design (FCRD) with eight replications having 50 seeds per replication. There were two factors considered in this experiment, factor A as soaking duration (S), factor B as incubation period (I) and dry seed as absolute control. Uniformly graded ridge gourd seeds were taken for standardizing the soaking duration and incubation period. The seeds were soaked in distilled water at $1: 1$ ratio on a volume basis for different duration's viz., $\left(S_{0}=\right.$ without soaking, $S_{1}=6 \mathrm{~h}, S_{2}=9 \mathrm{~h}, \mathrm{~S}_{3}=12$ $\mathrm{h}, \mathrm{S}_{4}=16 \mathrm{~h}, \mathrm{~S}_{5}=20 \mathrm{~h}$ and $\mathrm{S}_{6}=24 \mathrm{~h}$ ) at room temperature. After the specified period of soaking, the seeds were placed in between the moist gunny bags and incubated under dark condition at different days viz., ( $I_{0}=$ without incubation, $I_{1}=1$ day, $I_{2}=2$ days, $I_{3}=3$ days and $I_{4}=4$ days) to enhance radicle protrusion. The moisture content of the gunny bags is maintained by sprinkling water whenever needed. To know the effect of soaking alone, seeds were soaked in distilled water for the above-mentioned durations and used for conducting germination test without keeping for incubation. Similarly seeds without soaking, but incubated in the moist gunny bags was also included in the germination test to know the effect of incubation. Data were recorded for parameters viz., sprouting percentage, germination percentage, root length, shoot length, dry matter production and vigor index I \&ll to know the effect of the treatment on pre-germination of seeds.

\section{Sprouting percentage}

The sprouting percentage was calculated for seeds kept in the incubation by counting the number of radicles protruded seeds. Observations on radicle 
protrusion were taken on hourly intervals and the seeds showing radicle protrusion were counted daily from the first day after incubation. Those sprouted seeds were used for conducting germination test in sand medium immediately, until the completion of sprouting. The seeds were considered as germinated when the radicle length is between $1 \mathrm{~mm}$ to $3 \mathrm{~mm}$ were selected for conducting the germination test. The sprouting percentage was calculated based on the number of seeds sprouted on each day to the number of seeds kept for incubation, and the results were expressed in percentage.

\section{Germination percentage}

After incubation, the germination test was carried out with the pre-germinated seeds from each replication and combinations in a sand medium based on the procedure explained by ISTA (2012). The pre-germinated seeds with optimum radicle length were placed at an equal distance with uniform depth in sand-filled trays and then the germination test was carried out at optimum condition (25 \pm $2^{\circ} \mathrm{C}$ temperature \& $95 \pm 5^{\circ} \mathrm{C}$ per cent relative humidity with uniform light) in the germination room. Germination test was also conducted for control seeds (dry seeds, water-soaked seeds without incubation and incubated seeds without soaking). The final count was taken on the $14^{\text {th }}$ day as per the Indian Minimum Seed Certification Standard (IMSCS). On the day of final count, the number of normal seedlings (seedlings showing normal root and shoot development) to the total seeds placed in the germination test was calculated, and the mean value was expressed as germination percentage.

\section{Root length}

Ten normal seedlings were randomly selected from the seedlings used for calculating germination percentage and the length of the root is measured from the collar region to the tip of the root that is expressed in centimeter.

\section{Shoot length}

Ten normal seedlings, and the length of the shoot was measured from the collar region to the tip of the primary leaf, that is expressed in centimeter.

\section{Dry matter production}

Ten normal seedlings selected for root and shoot length measurement were kept inside a brown paper cover and shade dried for $24 \mathrm{~h}$, then it is dried in a hot air oven maintained at $80^{\circ} \mathrm{C}$ for $24 \mathrm{~h}$. After oven drying, the dried samples were cooled in a desiccator for 30 minutes and weighed. The dry weight per seedling was expressed in g per 10 seedlings.

\section{Vigor index I (Abdul - Baki and Anderson, 1973)}

The vigor index I of the seedling was computed by multiplying germination percentage with the seedling length (sum of root length and shoot length) and expressed in whole numbers.

Vigor index I = Germination (\%) $x$ Seedling length $(\mathrm{cm})$

\section{Vigor index II (Abdul - Baki and Anderson, 1973)}

The vigor index II of the seedling was calculated by multiplying germination percentage with dry matter production and expressed in whole numbers.

Vigor index II = Germination (\%) $x$ Dry matter production

\section{Statistical analysis}

The data collected with respect to the various parameters observed were analyzed statistically for significance and the normality of data was checked. Non-normal data were transformed using arcsine transformation for percentage data like sprouting and germination before analysis, as suggested by Gomez and Gomez (1984). Experimental data were analyzed using IBM SPSS statistics software version 22 , and Duncan's multiple range test at the $5 \%$ level was used to compare the means of the treatments. Graphical representations of data were made using Microsoft Excel program.

\section{RESULTS AND DISCUSSION}

Based on the results obtained from the study, it was observed that there is a significant difference among the treatments (at 5\% probability level) on various parameters of ridge gourd seed. The results showed that $20 \mathrm{~h}$ soaking with one-day incubation $\left(S_{5} I_{1}\right)$ in dark condition proved superior in all parameters when compared with other treatments. Seeds without soaking but incubated recorded results similar to that of absolute control. On the other hand, seeds soaked but without incubation performed better than absolute control and seeds without soaking in all parameters. This may be due to the positive effect of soaking on seeds which is supported by Shahzad et al. (2003), who recorded earlier and higher germination when the rice seeds are soaked in water for 24 h.The pre-germination technique was found to suitable for breaking physical dormancy of seeds because, for pre-germinating the seeds, soaking followed by incubation is practiced in which, the seeds were soaked for the prescribed duration, that activates the hydrolytic enzymes and initiates the pre-germinative metabolism. After soaking, seeds were incubated in dark conditions for enhancing the radicle protrusion. The radicle protrusion is rapid and fast than normal dry seed because, during soaking, it softens the hard seed coat, which makes the radicle protrude easily. Because of all these reasons, the pre-germination technique was found to be more suitable for releasing physical dormancy of gourds and some of the tree seeds.

The recorded data and results after analysis 
on various parameters viz., Sprouting percentage, Germination percentage, root length, shoot length, Dry matter production, vigor index I \& II, and the results obtained after statistical analysis is given below.

\section{Sprouting percentage}

Sprouting percentage of seeds varied significantly due to soaking duration and incubation period on ridge gourd seed. Among the treatments taken, seeds soaked for $20 \mathrm{~h}$ with one-day incubation $\left(\mathrm{S}_{5} \mathrm{I}_{1}\right)$ have significantly recorded the highest sprouting (75\%) on the first day when compared with other soaking and incubation periods (Figure 1).

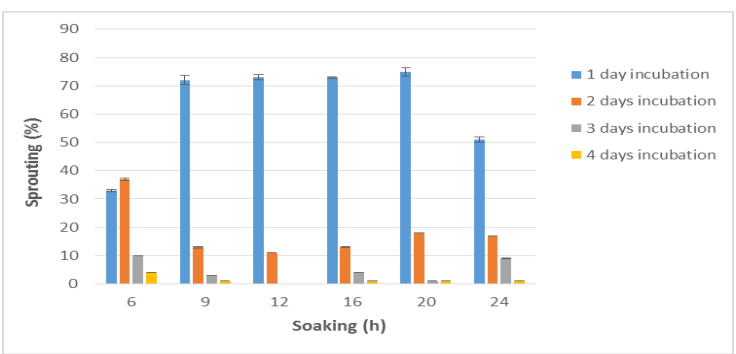

Figure 1. Effect of soaking duration and incubation period on sprouting percentage of ridge gourd var. PKM 1

A similar study was also conducted in bitter gourd seed in which, the pre-germinated seeds resulted in rapid and uniform germination with better seedling emergence (Thirusenduraselvi and Jerlin, 2010).

In cucurbits, Malik et al. (2001) found uniform growth in all plants, when pre-germinated seeds were used for planting. During incubation, the temperature inside the chamber was found to be increased from $30^{\circ} \mathrm{C}$ to $32^{\circ} \mathrm{C}$ that is mainly because of the heat produced by the seed during respiration process. Using this heat and dark condition, the enzyme gets activated, and radicle protrudes earlier than normal dry seeds used for germination.

The moisture content of the gunny bags prevents the seed from drying and softens the seed coat even after soaking which removes the mechanical barrier and allows the radicle to protrude easily. The incubation period can be extended up to 2 days as there is a considerable amount of sprouting was observed. The incubation period should not be extended for more than two days, because it may reduce the germination percentage.

The reason is, as the radicle length will become more than $3 \mathrm{~mm}$ when continuous incubation was done, it will produce abnormal seedlings when used for conducting the germination test. This was supported by the findings of Gray (1977), who reported that sowing pre-germinated seeds having radicles longer than $2 \mathrm{~mm}$ gave more variable levels of emergence and often reduced emergence, particularly when they were sown into dry or drying seed beds.

\section{Germination percentage}

The treatment combination $20 \mathrm{~h}$ soaking with one-day incubation $\left(\mathrm{S}_{5} \mathrm{I}_{1}\right)$ had higher germination (96\%) than other treatment combinations in this study. Whereas, the lower germination (74\%) was observed in absolute control i.e., dry seed (Figure 2).

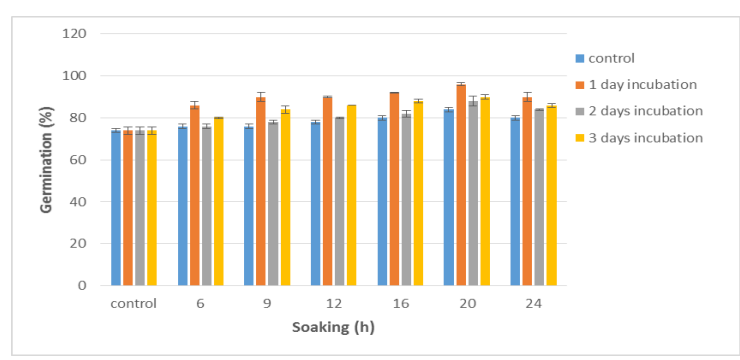

Figure 2. Effect of soaking duration and incubation period on germination percentage of ridge gourd var. PKM 1

It might be effective because of softening of hard seed coat during soaking which makes the radicle to come out by overcoming mechanical restrictions.

This is mainly followed in slowly germinating crop seeds due to hard seed coat, which are regularly pre-germinated in addition to pre-soaking, thereby the growth of the germ root is already started before the seed is sown into the field or portrays. This will still cause quicker emergence of the seeds sowed and a lesser risk of damage by diseases, pests or weather conditions.

Bichi (2012) who elucidated that, soaking seeds in water increases the germination as well as fastens the seedling emergence by increasing the hydrolytic enzyme activity and supported the effect of water soaking on germination of seeds. The effect of pregermination was explained by Meena et al. (2013) in wheat and reported that Hydro-primed and pregerminated seeds emerged earlier and faster than dry seeds leading to better crop establishment, higher tillering and grain yield.

\section{Shoot length and root length $(\mathrm{cm})$}

Pre-germinated seeds had significantly produced seedlings with the highest shoot and root length than control seeds. $20 \mathrm{~h}$ soaked with one day incubated seeds $\left(S_{5} I_{1}\right)$ gave the highest length of the shoot $(20 \mathrm{~cm})$ and root $(17.1 \mathrm{~cm})$ whereas the lowest shoot length $(13.0 \mathrm{~cm})$ and root length $(12.5 \mathrm{~cm})$ was documented by control (dry) seed (Figure 3 and 4).

The increased shoot and root length maybe because of its early emergence than other seeds. This finding is in accordance with (Chrimes and Gray, 1982) who found that the pre-germinated lettuce seeds when sown reliably emerged earlier 
then pelleted or dry seeds. The improved seedling length might also be due to increased and rapid cell division within the apical meristem of shoot and root.

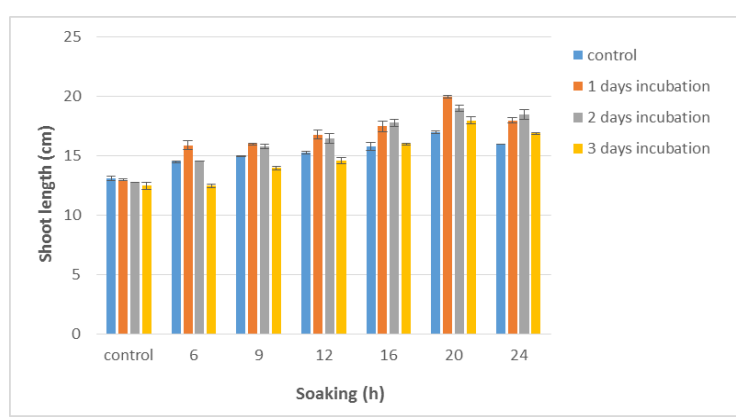

Figure 3. Effect of soaking duration and incubation period on shoot length of ridge gourd var. PKM 1

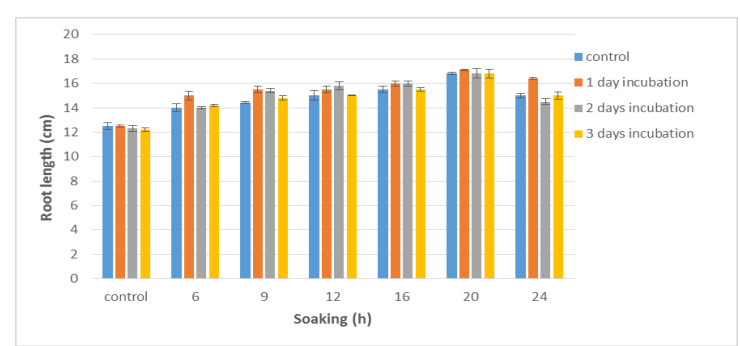

Figure 4. Effect of soaking duration and incubation period on root length of ridge gourd var. PKM 1

\section{Dry matter production (g/ 10 seedlings)}

The results obtained showed that there is a significant difference between the treatments. $20 \mathrm{~h}$ soaked and one day incubated seeds $\left(\mathrm{S}_{5} \mathrm{I}_{1}\right)$ recorded higher DMP (1.473 g), and the lowest (1.067 g) was recorded by the control (dry seed), which is explained in (Figure 5).

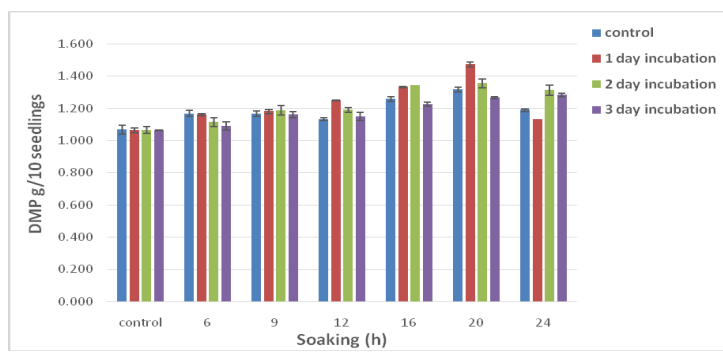

Figure 5. Effect of soaking duration and incubation period on dry matter production of ridge gourd var. PKM 1

The increased DMP might be due to the highest root and shoot length obtained by pre-germinated seeds then control seed. The findings are in agreement with (Ghate and Phatak, 1982) who found that faster emergence and heavier seedlings were observed in pre-germinated pepper seeds when compared with non-treated seeds

\section{Vigor index I and II}

Vigor index of pre-germinated seeds highly differed from the control seed. As the main objective of this study was to separate highly vigorous and viable seed in the seed lot the treatment that showed high vigor index was selected as the best treatment. $20 \mathrm{~h}$ soaked with 1 day incubated seeds $\left(S_{5} I_{1}\right)$ recorded maximum in both vigor index I (Figure 6), and II (Figure 7).

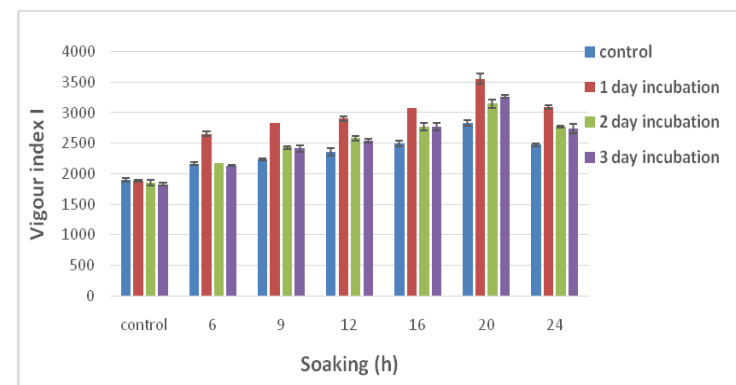

Figure 6. Effect of soaking duration and incubation period on vigor index I of ridge gourd var.PKM1

The highest vigor index I of 3562, and II of 149 was recorded by pre-germinated seeds and the lowest vigor index I of 1894 and II of 79 was recorded by control respectively. This might be due to the reason that pre-germinated seeds produced longer and stronger seedlings than dry seeds, which results in high vigor index of the seedlings.

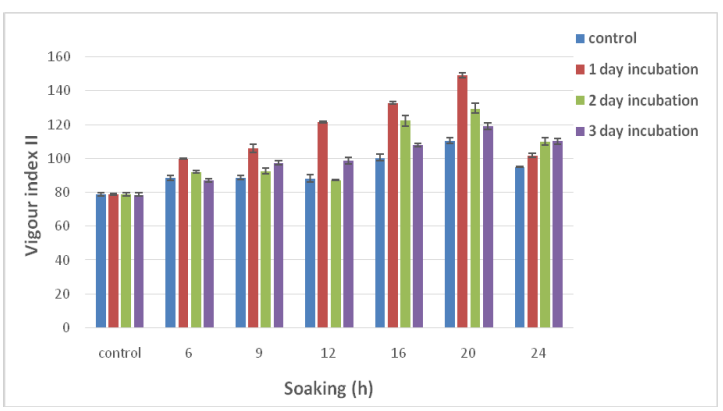

Figure 7. Effect of soaking duration and incubation period on vigor index II of ridge gourd var.PKM1

The radicle emergence of the seed is directly related to the vigor status of the seeds i.e., high vigor seeds sprouted earlier compared with low vigor seeds. This fact was supported by Chinnasamy and Sundareswaran (2019), who found that, vigorous seeds can be separated based on radicle emergence time and its length in cluster bean seed lot to get good field emergence.

\section{CONCLUSION}

Gourds normally exhibit hard seed coat, which restricts the seed germination and results in uneven germination of seeds. To overcome this problem pre-germinating the seeds before sowing can be practiced. Results obtained in the experiment reveals that soaking seeds in distilled water for $20 \mathrm{~h}$ followed by one-day incubation recorded the highest value for all parameters that can be practiced to overcome germination related problems in ridge 
gourd seeds. This technique can be used in cases where farmers are having limited resources (e.g., capital) to go for high-cost chemical or technology as the cost involved in this treatment is zero except the seed cost. This technique can be adapted to overcome problems in ridge gourd cultivation before sowing to remove the dead and low vigor seeds, which in turn will help to maintain the optimum plant population in the field to get optimum yield.

\section{REFERENCES}

Abdul-Baki, A.A. and J.D. Anderson. 1973.Vigor determination in soybean seed by multiple criteria 1. Crop science., 13(6): 630-633.

Bates, D.M., Robinson, R.W., and C. Jeffrey. 1990. An outline of the Cucurbitaceae. Biology and Utilization of Cucurbitaceae., 449-463.

Bichi and M. Aminu. 2012. Different pre-germination treatments and Delonix regia seeds. Jorind 10(2): 24-29.

Bidhan, R. 2013. Innovative technique for enhancement of seed germination and seedling establishment of cucurbits (Cucumis sativus L.). International journal of agricultural sciences., 9(2): 663-666.

Burkill, H.M. (1985). The Useful Plants of West Tropical Africa, 2nd edn. 1: Families $A-D$. Royal Botanic Gardens, Kew, U.K., 960pp.

Bruggink, G.T., and Van der Toorn, P. 1996. U.S. Patent No. 5,522,907. Washington, DC: U.S. Patent and Trademark Office.

Chinnasamy, G.P. and S.Sundareswaran. 2019. Evaluation of radicle emergence test to predict seed vigor and field emergence in different seed lots of cluster bean (Cyamopsis tetragonoloba L.). Madras Agric. J., 106(spl): 143-149

Chrimes, J.R. and D. Gray. 1981. Comparisons of the use of pre-germinated, dry and pelleted seeds for block-raising of glasshouse lettuce.Scientia Horticulturae., 17: 15-25

Fernando, L.N. and I.U. Grun. 2001. Headspace-SPME analysis of volatiles of the ridge gourd (Luffa acutangula) and bitter gourd (Momordica charantia) flowers. Flavour and Fragrance Journal, 16(4): 289-293.
Ghate, S.R. and S.C. Phatak. 1982. Preference of tomato and pepper seed germinated before planting. J. Amer. Soc. Hort. Sci., 107: 908-911.

Gomez., A. Kwanchai and Arturo A Gomez. 1984. Statistical procedures for agricultural research: John Wiley \& Sons.

Gray, D. 1977. Temperature sensitive phases in the germination of lettuce (Lactuca sativa) seeds. Ann. Appl. Biol., 86: 77-86.

Gray, D. 1981 . Fluid drilling of vegetable seeds. Hort. Rev, 3: 1-27.

ISTA. 2012. International Rules for Seed Testing. Bassersdorf, Switzerland.

Krishnasamy, V. 1991. Effect of sowing pre-germinated seeds on seedling emergence in bittergourd. Seed science and technology 19(2): 227-233.

Lim, T.K. 2012a. Cucumis metuliferus. In: Edible Medicinal and Non-Medicinal Plants.Springer, Dordrecht, the Netherlands, pp. 235-238.

Lim, T. K. 2012b. Sechium edule. In: Edible Medicinal and Non-Medicinal Plants. Springer, Dordrecht, the Netherlands, pp. 384-391.

Malik, Inder Jeet, T.L. Ellington, T.C. Wehner, and D.C. Sanders. 2001. Seed treatment effects on emergence of Luffa sponge gourd. REPORTCUCURBIT GENETICS COOPERATIVE 24: 107-109.

Meena, R.P., Sendhil, R., Tripathi, S.C., Chander, S., Chhokar, R.S. and R.K. Sharma. (2013). Hydropriming of seed improves the water use efficiency, grain yield and net economic return of wheat under different moisture regimes.SAARC Journal of Agriculture., 11(2): 149-159.

Shahzad M.A.B., Muhammad. F. and K. Abdul. 2003. Comparative study of pre-sowing seed enhancement treatments in fine rice (Oryza sativa L.). Pakistan Journal of Life and Social Science., 1(1): 21-25.

Thirusenduraselvi, D, and R Jerlin. 2010. Effect of pre-germination treatments on the emergence percentage of bitter gourd cv. CO 1 seeds. Tropical Agricultural Research and Extension 10: 88-90.

Uriostegui Arias, M. T. 2015. Phytochemical analysis and antiproliferative effect of genotypes S. edule (Jacq.) Sw. On breast cancer. A Thesis (MSc, specialist in Botany), University of Mexico. 